\title{
Gobernanza y políticas de intervención en espacios públicos de Ciudad Juárez.
}

\author{
Juan Enrique Diaz"
}

La gestión realizada por una asociación civil en la colonia Oasis Revolución hizo legítima la estrategia de política pública de intervención urbana Todos somos Juárez como un proceso aislado de aparente gobernanza para acallar las demandas sociales de empleo y equipamiento urbano en el marco de la desindustrialización de maquilas y la guerra contra el narcotráfico en Ciudad Juárez, Chihuahua.

Los problemas urbanos no responden a soluciones técnicas, responden a soluciones políticas.

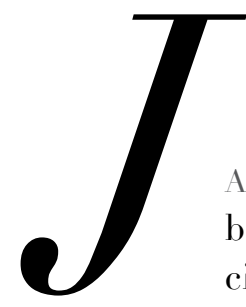

AN BAZANT' EXPLICA la planificación urbana como un instrumento de regulación y cambio social, asimismo, como un elemento articulador en la relación entre gobierno y gobernados en la ciudad, en los espacios en que participan sus habitantes. Este vínculo se basa en una metodología donde la importancia de un proceso multidisciplinar de gestión en la aplicación

\footnotetext{
* Presidente del Centro de Investigación para el Desarrollo Urbano y Comunitario A.C. y docente del Instituto de Arquitectura, Diseño y Arte de la Universidad Autónoma de Ciudad Juárez.
}

entre técnicos, ciudadanos y gobierno ayuda a generar determinados planes para un centro urbano o espacio público.

Entender la planificación urbana implica conocer los procesos políticos, el análisis en la aplicación de políticas públicas y la forma en que éstas se diseñan. Para ello, es importante analizar el diseño de políticas públicas y cómo se han aplicado en los procesos urbanos, principalmente en la etapa inicial. Las etapas de análisis concentran las dinámicas en que se han desarrollado diversas intervenciones urbanas. Conocer ese proceso y comprender cómo lo vivieron sus actores nos ayuda a entender la problemática urbana con mayor profundidad y valorar la forma en que se atendió. 
El presente trabajo se basa en propuestas de intervención urbana del aparato gubernamental en el espacio público con la participación institucional y ciudadana. Se revisa la participación ciudadana con el Estado en Ciudad Juárez, Chihuahua, a partir de un estudio de caso, donde se analizará el diseño de políticas públicas para realizar intervenciones urbanas en espacios públicos y la coordinación para llevarlas a cabo. Para el caso específico se consideraron las intervenciones de 20 Io contenidas en la estrategia Todos somos Juárez.' Para elegir el estudio de caso se visualizaron la mixtura de actores participantes con mayor representatividad, así se eligió la intervención urbana en el sector de Oasis Revolución. En la tabla i se observan las intervenciones realizadas y la que contenía una mayor diversidad y mejor alcance por parte de actores participantes y por la información documental obtenida.
Gobernanza y PROCESO METODOLÓgico

El desarrollo de este espacio muestra un panorama e identifica a actores por relevancia y carácter contextual aproximándose más a un proceso de gobernanza que muestra mayor riqueza entre ellos y una mayor variedad de actores y relaciones a partir de su participación. Una vez identificado el estudio de caso y delimitada la investigación, la idea general de abordar el proceso fenomenológico a través de las teorías de las políticas públicas, es valorar la forma en que los actores participan y se desarrollan dentro de este proceso mismo, valorando esta relación entre los diversos actores participantes y cómo perciben el enfoque de jerarquías, así como dinámicas entre la sociedad abierta y el gobierno.

Este precepto se entiende como una aproximación a la nueva gestión o gobernanza de Luis Aguilar, ${ }^{3}$ donde el gobierno actúa acompañado de diversos actores

TABLA I

Intervenciones con recursos del programa Todos somos Juárez

\begin{tabular}{|c|c|c|c|c|c|}
\hline \multirow{2}{*}{ Intervención urbana } & \multirow{2}{*}{ Colonia } & \multicolumn{2}{|c|}{ Tipo } & \multicolumn{2}{|l|}{ Ejecución } \\
\hline & & Social & Físico & Social & Físico \\
\hline Parajes del Sur & $\begin{array}{l}\text { Parajes del } \\
\text { Sur }\end{array}$ & $X$ & & $\begin{array}{c}\text { Universidad Autónoma de Ciudad } \\
\text { Juárez (UACJ) }\end{array}$ & $\begin{array}{l}\text { Secretaría de Desarrollo } \\
\text { Social (Sedesol) }\end{array}$ \\
\hline Parque Oasis & $\begin{array}{l}\text { Oasis } \\
\text { Revolución }\end{array}$ & $\mathrm{X}$ & $\mathrm{X}$ & $\begin{array}{l}\text { Fomento del Tejido Social } \\
\text { de Ciudad Juárez (Foteso) }\end{array}$ & $\begin{array}{c}\text { Ayuntamiento de Ciudad } \\
\text { Juárez-Sedesol }\end{array}$ \\
\hline Parque Clouthier & $\begin{array}{l}\text { Manuel J. } \\
\text { Clouthier }\end{array}$ & $X$ & & $\begin{array}{c}\text { Instituto Municipal de } \\
\text { Investigación y Planeación de } \\
\text { Ciudad Juárez (IMIP) }\end{array}$ & Sedesol \\
\hline PEC Alto & $\begin{array}{l}\text { Plutarco E. } \\
\text { Calles }\end{array}$ & $X$ & $\mathrm{X}$ & UACJ & Municipio- Sedesol \\
\hline Cancha femenil & $\begin{array}{l}\text { Plutarco E. } \\
\text { Calles }\end{array}$ & $\mathrm{X}$ & & UACJ & Sedesol \\
\hline Granito & Morelos & $X$ & & IMIP & Sedesol \\
\hline Km. 29 & Kilómetro 29 & $\mathrm{X}$ & & IMIP E-I & Sedesol \\
\hline Francisco Villareal & $\begin{array}{l}\text { Tierra Nueva } \\
\text { Etapa I }\end{array}$ & $\mathrm{X}$ & $\mathrm{X}$ & UACJ & Sedesol \\
\hline
\end{tabular}




\begin{tabular}{|c|c|c|}
\hline Determinación & Contenido & Seguimiento \\
\hline
\end{tabular}

Etapas en la política pública

\begin{tabular}{ccc}
\hline Agenda & Decisión e Implementación & Evaluación \\
\hline $\begin{array}{c}\text { Posibles indicadores para la realización de técnicas de investigación } \\
\text { Problemática, jerarquía, homologación } \\
\begin{array}{c}\text { de agendas, agenda pública, agenda } \\
\text { privada, coordinación. }\end{array}\end{array}$ & $\begin{array}{c}\text { Estrategias, intervenciones, diseño } \\
\text { de proyectos, gestiones, } \\
\text { materialización, coordinación }\end{array}$ & $\begin{array}{c}\text { Programas de continuidad, } \\
\text { formulación de seguimiento, } \\
\text { regulación, coordinación }\end{array}$ \\
\hline
\end{tabular}

Fuente: Elaborado a partir de Hernández (2006).

que le proveen instrumentos y formas posibles de entender mejor las problemáticas partiendo de la sociedad misma, convirtiéndose en agente y actor del proceso de toma de decisiones, implementaciones y evaluaciones de una política pública generada para mejorar la realidad de determinado sector.

Bajo este enfoque se filtraron las categorías propuestas en el marco teórico sobre el análisis de las políticas públicas de Parsons. ${ }^{4}$ Así, se cuenta con tres principales categorías definidas: determinación, contenido y seguimiento de las políticas públicas. Dentro de éstas se encuentran las etapas respectivas del ciclo: agenda, decisión, acción, implementación y evaluación.

A través de estas categorías se valora cómo se involucraron los actores y agentes en esta dinámica a través del diseño de la política pública implementada, así como las relaciones que se desarrollaron con los diversos actores participantes para mostrar la conceptualización de gobernanza durante el periodo. Se analizaron los documentos y los reportes emitidos por las diferentes instituciones y sociedades, así como información documental hemerográfica de eventos durante el desarrollo de la intervención, mismos que ofrecerán datos relevantes del proceso. También se analizó la opinión de sus participantes en el proceso, se elaboraron entrevistas entre los actores y agentes, incluyendo actores clave en el desarrollo de la intervención, para saber cómo fue su participación en este proceso y de qué forma lo hicieron y si se dieron las condiciones de un entendimiento mutuo entre la sociedad y el gobierno.

Las entrevistas son para valorar cómo el fenómeno mismo que contiene sus vivencias y relatos y acciones se dieron durante el proceso; a partir de los actores mismos, las entrevistas se contrastan con las categorías, etapas y posibles indicadores para permitir que el actor exprese cómo fue el proceso de participación propio; se llevaron a cabo de forma semiestructurada y a profundidad a determinados actores donde se percibió la participación de los demás agentes y usuarios respecto a la intervención realizada; y se partió de las etapas mismas de las que se conoció cómo se llevaron a cabo entre los actores participantes las siguientes categorías (véase tabla 2).

La intención es mostrar los resultados de la etapa de la determinación de la política pública aplicada, misma que al ser la fase inicial dicta el rumbo a partir de la mixtura de actores participantes e instituciones para mostrar los niveles de inclusión de la política pretendida.

La determinación refiere cómo se integraron las agendas tanto públicas como de gobierno, cómo se valoran y jerarquizan las demandas ciudadanas sobre situaciones o rezagos que se requiere atender y cuáles 
fueron tomadas en cuenta, es decir, una problemática que se intenta transformar; en esta etapa podemos discernir ciertos indicadores tales como la jerarquía de la atención de la problemática.

La agenda se basa en diversas categorías: ¿cómo discernir qué asuntos tienen el potencial para ser tomados en cuenta por una agenda pública?, ¿los sistemas políticos deben intervenir en la depuración, clasificación y anticipación de los asuntos?, y, si intervienen, ¿sus razones son sustentables y sus procedimientos son legales e imparciales? ${ }^{9}$ Cabrero y García ${ }^{7}$ identifican tres escenarios de agenda en México: el tradicional, que se basa en procesos comúnmente contenidos en el presupuesto local, donde se plantean problemas básicos como infraestructura y se manifiesta de forma reactiva; el ampliado, que maneja tanto problemas básicos como el tradicional, pero de tipo provisorio, incorporando estrategias para entender problemas como la planeación urbana o la zonificación, procurando recursos fuera del presupuesto local; y el integral, el cual maneja conceptos de de- sarrollo integral, competitividad, así como bienestar social y ambiental, entre otros. Cabrero y García ${ }^{8}$ identifican esta agenda como proactiva y plantean la participación de gobiernos, redes ciudadanas, redes intergubernamentales e internacionales, ampliando así su marco de ingresos y sus fuentes de financiamiento.

\section{Sector OAsis Revolución: ESTUDIO DE CASO}

La colonia Oasis Revolución es de relativa reciente creación y posee las características de los nuevos espacios habitacionales creados después de la consolidación manufacturera. Está ubicada al sur-poniente de la ciudad (véase figura I). El fraccionamiento fue autorizado en ig88 con un total de 3,o8 I lotes, con dimensiones de 7.0 metros de frente y $\mathbf{7} .50$ de fondo, dando una superficie de $122.5 \mathrm{~m}^{2}$.

\section{Ubicación de la colonia Oasis Revolución en la mancha urbana de Ciudad Juárez}

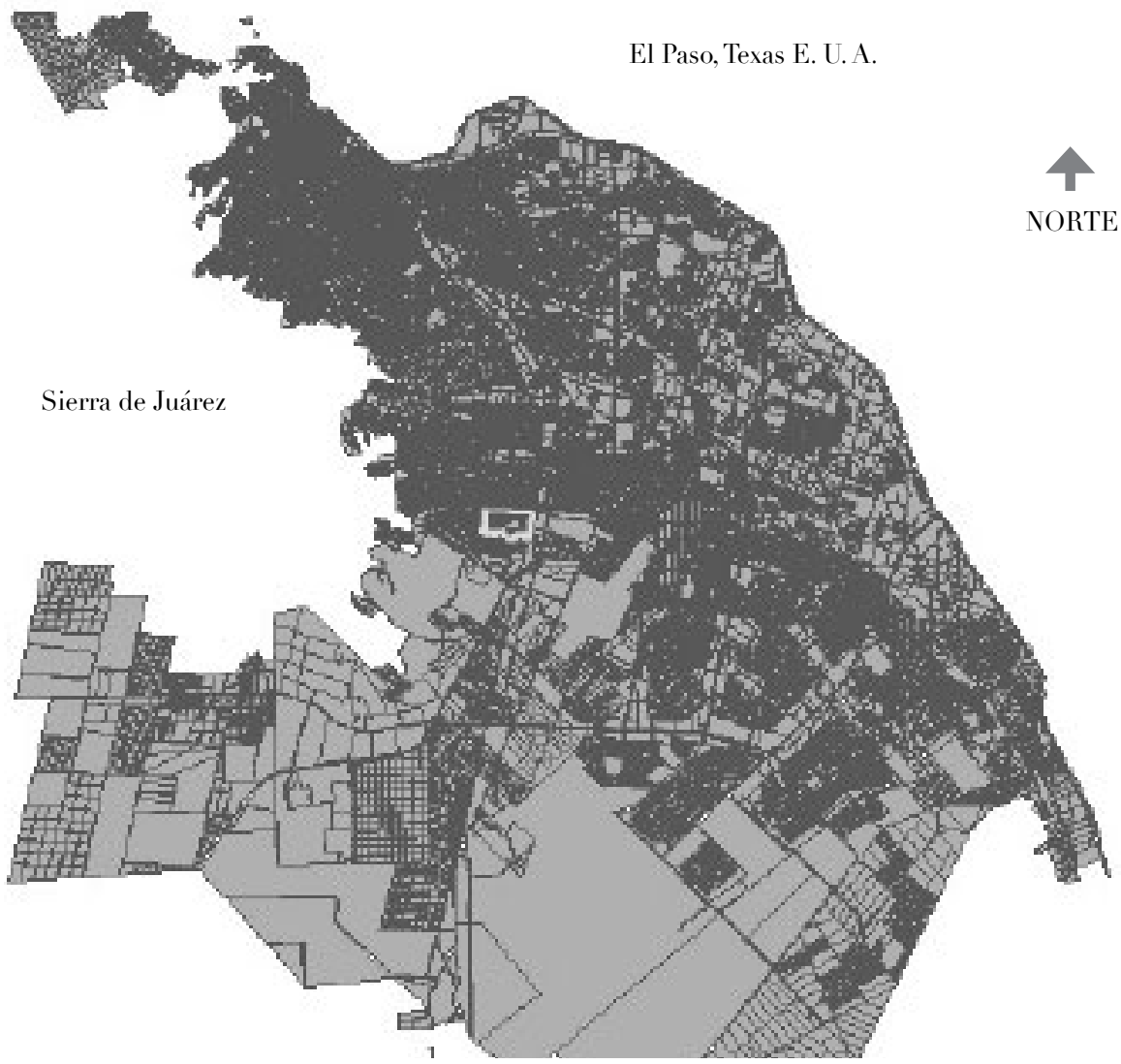

Fuente: Elaborado a partir de IMIP, 20I0. 
El sector era dinamizado por los parques industriales ubicados alrededor. La estrategia de crear estos sectores habitacionales básicamente se concentraba en dar vivienda a los trabajadores de la industria de manufactura creciente en el sector. La colonia se consolidó debido al creciente número y capacidad de ocupación de parques industriales ubicados en el sur de la ciudad en la década de los noventa; entre éstos se encuentra el más cercano del sector, el Parque Industrial North Gate, el Parque Industrial Aeropuerto, Aeroparque Industrial Verde, el Parque Industrial Zaragoza y fábricas de manufactura individuales fuera de éstos, parques industriales que se encuentran en el sector, sumando cerca de 9 mil plazas laborales. ${ }^{9}$

Dentro de la colonia se encuentra el espacio público destinado a equipamiento urbano, que se encontraba en desuso y deterioro, un terreno de $36 \mathrm{mil} \mathrm{m}^{2}$ que sirvió en un principio para las primeras obras de mejora, unas canchas de futbol, realizadas por una asociación civil (Fomento del Tejido Social de Ciudad Juárez, A. C. -Foteso-) y habitantes del sector.

El fraccionamiento se desarrolló como la mayoría de los construidos en la época del auge industrial, cerca de grandes parques industriales; con el tiempo manifestó rezago en diversos rubros de desarrollo urbano. La falta de equipamiento y de espacios para desarrollo social generó problemáticas encontradas previo a la violencia y drogadicción en la ciudad, siendo el fraccionamiento Oasis Revolución uno de los de mayor relevancia.

\section{GobernaNZA EN INTERVENCIÓN}

DEL ESPACIO PÚBLICO

La gobernanza se orienta, según Aguilar, ${ }^{10}$ en una acción que busca orden institucional y técnico, es decir, un proceso directivo estructurado de forma institucional y técnica, dándole eficacia a la administración pública y a las acciones gubernamentales. $\mathrm{Al}$ complementar con ayuda de la iniciativa privada, académica, el mercado y la sociedad, y considerando la mención de Parsons" de que los bienes públicos puros son de injerencia del Estado, se forma un entramado de redes que influyen en un proceso de buen gobierno, donde se encuentra el equilibrio entre sus participantes.

El objetivo principal de las respuestas sobre cada etapa del proceso, por parte de los actores participantes, es profundizar cómo se llevó a cabo y cómo se interactuó con los demás actores de las diferentes insti- tuciones. De esta forma, se obtuvieron las vivencias de los actores sin importar el resultado al darle relevancia al proceso mismo en sentido de gobernanza sobre la coordinación y las interrelaciones entre el gobierno, instituciones civiles o privadas y sociedad.

\section{Análisis de la "DETERminación” DE LA POLítica PÚBLICA APLICADA}

Las principales variables de la agenda se determinan en su formación, es decir, si es de gobierno o es pública. Esto genera de entrada una distancia entre ambas, sobre todo si se desea que los gobiernos empaten sus agendas con las necesidades de los habitantes. La agenda que se obtuvo inicialmente obedeció a una agenda pública local, que se convirtió en agenda de gobierno al estructurarse la estrategia Todos somos Juárez.

De acuerdo con la información recabada, se mostraron los intereses por parte de las instituciones. El Gobierno Federal manifestó el interés de generar proyectos de acuerdo con el contexto y realidad de Ciudad Juárez. El programa Todos somos Juárez intentó una aproximación a trabajar en conjunto con la sociedad de la ciudad, inicialmente a través del documento redactado para generar y estructurar la intervención del programa:

- "El Gobierno Federal reconoce que los juarenses son quienes mejor conocen la situación de su ciudad y quienes más pueden hacer para resolver la problemática que los aqueja."

- "El Gobierno Federal comprendió que el problema de Juárez era el tejido social y Fomento del Tejido Social de Ciudad Juárez (Foteso A. C.) lo había señalado cuatro años antes, sumándose a la estrategia legitimada por la comunidad." ${ }^{3}$

- "Participamos en la agenda la Dirección del programa de rescate de espacios públicos y la coordinadora nacional de los proyectos sociales así mismo la comunidad." "14

- "Fue muy difícil empezar aquí, hacer que la gente entienda y participe es un trabajo complicado y lento, así que nos costó mucho trabajo realizar todo esto que está ya palpable.”̌็

- "Intentamos participar en la mesa de la Secretaría de Desarrollo Social (Sedesol) pero la mafia encabezada por dirigentes de la mesa bloqueó nuestro intento y el de muchas otras organizaciones no 
gubernamentales $(\mathrm{ONG})$, ya que no aceptaron que pudiéramos si quiera tener voz en esa mesa." ${ }^{6} 6$

- "La intención era crear una intervención en la mesa de desarrollo social a nivel ciudad y no que se destinaran los recursos a sólo tres espacios públicos, entre éstos el de Oasis Revolución, todos querían algo."

- "Obtuvimos en comodato el predio abandonado y lleno de basura, animales muertos y contaminación que por aquel entonces era el terreno destinado al parque Oasis Revolución.”.18

- "Es bueno tener el parque, ya que era un sitio muy inseguro antes que lo arreglaran los de la iglesia." ${ }^{9}$

- "Ellos tenían el parque para cosas de la iglesia, lo usaban como estacionamiento, para las misas."

- "Los principales tipos de servicios que demanda la población en los espacios públicos, en orden de importancia son: las actividades deportivas, capacitación en oficios, salud, culturales y recreativos."

- "Contar con un parque con juegos y áreas deportivas, un lugar de reunión y convivencia vecinal, fueron los principales ejes del programa arquitectónico del proyecto y fueron presentados y validados por la comunidad." ${ }_{22}$

Retomando las tipologías de las agendas propuestas por Enrique Cabrero, ${ }^{23}$ este estudio de caso se aproxima a una agenda ampliada, considerando que los recursos son netamente de gobierno y la agenda no contempla otras fuentes para obtención de recursos. La propuesta de la agenda, debido a la incorporación de redes ciudadanas como la asociación civil, puede interpretarse hasta cierto punto como un acercamiento importante para entender los problemas públicos de este determinado sector. De esta forma se manifestaron las problemáticas y de acuerdo con entrevistas, se mantuvo cierta homologación entre las agendas, tanto pública del sector como de gobierno y de la forma en que la asociación civil ha articulado las necesidades de la colonia.

- "Las principales problemáticas detectadas fueron la delincuencia y la inseguridad en la mayoría de las colonias, en el caso de Oasis Revolución se tuvo un trabajo a mayor profundidad por parte de la asociación civil." ${ }^{24}$

- "Partimos del estudio realizado por la empresa Confirme en nuestra comunidad del r al ıo de mayo del 2006 donde se encontraron datos muy relevantes en las y los ciudadanos de nuestra colonia.” ${ }^{25}$
- El déficit de espacios públicos y equipamiento recreativo y deportivo para dar servicio a los habitantes, aunado a que estuviera en zonas de marginación." ${ }^{6}$

- Entrega del estudio a la comunidad en reuniones con puros padres de familia, con madres de familia como grupos específicos y de un modo especial con jóvenes, grupo al que se piensa destinar toda la acción, esfuerzo, trabajo y recursos, primero por ser mayoría y segundo por ser nuestra esperanza."’’7

- "Los de la parroquia han trabajado mucho para que esto esté funcionando y para que nosotros usemos este lugar y para que participemos con ellos para pedir más al gobierno". ${ }^{28}$

- "Han estado controlando el parque (los de la iglesia) y no han dejado que la gente entre a vender sus cosas, conozco gente que no ha podido entrar". ${ }^{29}$

Hasta cierto punto, el desarrollo de la agenda parecía complementarse dentro de las necesidades de los vecinos de la colonia, y las propuestas del gobierno fueron encaminadas con mayor facilidad de acuerdo con los actores entrevistados, debido a la presencia de la asociación civil y los datos que manejaba con anterioridad a la propuesta del Gobierno Federal: "El caso de Oasis Revolución simplificó la tarea, ya que con anterioridad habíamos estado trabajando con ellos algunos proyectos, inclusive específicos en el predio donde ahora se encuentra el espacio público realizado por Sedesol". ${ }^{30}$

De esta forma, la agenda inició con el estudio por parte de la asociación civil para seguir los esfuerzos por la adquisición del predio en comodato, realización de proyectos dentro de este predio, y después de aprovechar la coyuntura del programa federal, llevar a cabo las necesidades de los habitantes del sector, en su mayoría, encaminadas por la asociación civil.

- "Hasta la fecha se han cubierto la mayoría de las demandas y propuestas de la comunidad, sólo falta realizar la biblioteca."

- "El presupuesto no fue suficiente, ya que faltó la biblioteca del centro comunitario, una de las principales propuestas de la comunidad." ${ }^{2}$

\section{Reflexiones finales}

La asociación civil Fomento del Tejido Social de Ciudad Juárez e Instituto Municipal de Investigación y 
Planeación de Ciudad Juárez, apoyados por la Secretaría de Desarrollo Social, generaron propuestas y construyeron la agenda pública del sector Oasis Revolución sobre la cual se basaron los primeros proyectos, ya desarrollados, entre la asociación civil y el IMIP, previo a la estrategia Todos somos Juárez, la cual finalmente destinó los recursos para la realización de la intervención urbana.

La agenda fue parte fundamental en el proceso del diseño de una política pública al definir las problemáticas para los posibles campos de acción y sus lineamientos específicos. De acuerdo con las características de Cabrero, ${ }^{33}$ el proceso de gobernanza en el estudio de caso de Ciudad Juárez se cumplió en su totalidad al mostrar la dificultad de desarrollar una agenda de política pública integral al ser éste un caso muy específico en actores y temática, limitando los recursos al Gobierno Federal y ejes de acción.

No obstante, lo observado se delimitó a lo siguiente:

- Se construyó en torno a una política específica. La idea general tanto de la comunidad, asociación civil, Sedesol e investigaciones y proyectos propuestos por el IMIP se concentraron en el enfoque de desarrollo social, en sentido de generación de un parque, producto de un espacio de equipamiento en deterioro existente en el sector.

- Tiene una visión de tiempo limitada. Aunque la asociación civil llevaba tiempo trabajando para realizar la totalidad de la obra, el proyecto era por etapas: al incluirse a la estrategia Todos somos Juárez, se concretó en tiempo y forma de acuerdo con los compromisos que adquirieron con el Gobierno Federal al determinar esta estrategia con compromisos definidos en temporalidad.

- Recursos identificados y etiquetados. El Gobierno Federal fijó el monto que destinaría para cada área de desarrollo y Sedesol definió las ubicaciones de cada espacio determinado por los polígonos de pobreza; la gestión de la asociación civil se centró en incluir este espacio (Oasis Revolución).

- Objetivos precisos en el tiempo. Esta intervención ya mostraba avances en cuanto a la creación de actividades y participación con los habitantes del sector, al momento de incluirse en la agenda Todos somos Juárez tenía ya identificados sus objetivos principales y reales para llevarse a cabo.

\section{$-\operatorname{notas} \cdot-$}

' Bazant, Jean (2011), Planeación urbana estratégica, métodos y técnicas de análisis, México, Editorial Trillas.

2Secretaría de Desarrollo Social (2010), Todos somos Juárez, en http:/www.todossomosjuarez.gob.mx/estrategia/index.html

${ }^{3}$ Aguilar, Luis (2006), Gobernanza y gestión pública, México, Fondo de Cultura Económica.

4 Parsons, Wayne (2007), Políticas públicas: una introducción a la teoría y la práctica del análisis de políticas públicas, México, Flacso.

${ }^{5}$ Hernández, Roberto, Carlos Fernández-Collado y Pilar Baptista (2006), Metodología de la investigación, México, McGrawHill/Interamericana Editores.

${ }^{6}$ Aguilar, Luis (1993), Problemas públicos y agenda de gobierno, México, Porrúa.

7 Enrique Cabrero, y Carlos García (2010), "La agenda de políticas públicas en ciudades mexicanas durante el siglo xx: ¿Cien años de soledad municipal?", Estudios Demográficos y Urbanos, vol. 25, núm. I, p. 73 .

${ }^{8}$ Enrique Cabrero y Carlos García (2010), op. cit.

9 IMIP, "Análisis estructural del empleo laboral en Ciudad Juárez", IMPI.

ı Luis Aguilar (2006), op. cit.

"Wayne Parsons (2007), op. cit.

12 Sedesol (2010), op. cit.
${ }^{3}$ Reporte de Actividades de Fomento del Tejido Social de Ciudad Juárez A. C. (Foteso).

14 Entrevista a representante de Sedesol.

เ̌ Reporte de Actividades de Foteso A. C.

${ }^{16}$ Idem.

17 Entrevista a miembro dirigente de la Mesa de Desarrollo Social.

${ }^{18}$ Entrevista a representante de Foteso A. C.

${ }^{19}$ Entrevista a representante de IMIP.

${ }^{20}$ Idem.

${ }^{2}$ Sedesol (2010), Consulta Ciudadana 2010, Unidad de Programas de Atención de la Pobreza Urbana.

${ }^{22}$ Entrevista a representante de IMIP.

${ }^{23}$ Enrique Cabrero y Carlos García (2010), op. cit.

${ }^{24}$ Entrevista a representante de Sedesol.

${ }^{25}$ Reporte de Actividades de Foteso.

${ }^{26}$ Entrevista a representante de IMIP.

${ }^{27}$ Entrevista a representante de Foteso A. C.

${ }^{28}$ Entrevista a vecino de Oasis Revolución.

${ }^{29}$ Idem.

${ }^{30}$ Entrevista a representante de IMIP.

${ }^{3}$ Entrevista a representante de Foteso.

32. Idem.

${ }^{33}$ Enrique Cabrero y Carlos García (2010), op. cit. 